\title{
Distribución de los hallazgos de escorpiones en la Ciudad de Buenos Aires en el período 2001-2012 y sus implicancias sanitarias
}

\author{
Distribution of findings of scorpions in Buenos Aires city in \\ the period 2001-2012 and their sanitary implications
}

\author{
Dr. Guillermo Blanco ${ }^{a}$, Prof. Biol. Rodrigo D. Laskowicz ${ }^{b}$, Lic. Laura C. Lanarib, \\ Méd. Eduardo Scarlato ${ }^{c}$, Dr. Carlos Damin ${ }^{d}$, Dr. Ernesto H. de Titto ${ }^{e}$, Dr. Adolfo R. de Roodt t,e
}

a. Instituto de Estudios de la Inmunidad Humoral/Consejo Nacional de Investigaciones Científicas y Técnicas (CONICET), CABA

b. Área Investigación y Desarrollo, Instituto Nacional de Producción de Biológicos (INPB)Administración Nacional de Laboratorios e Institutos de Salud (ANLIS) “Dr. Carlos G. Malbrán", CABA.

c. Servicio de Toxicología del Hospital de Clínicas "José de San Martín", Universidad de Buenos Aires, CABA.

d. $1^{\text {ra }}$ Cátedra de Toxicología, Facultad de Medicina, Universidad de Buenos Aires, CABA.

e. Dirección Nacional de Determinantes de la Salud e Investigación, Ministerio de Salud de la Nación, Ciudad Autónoma de Buenos Aires.

Correspondencia: Dr. Adolfo R. de Roodt, aderoodt@gmail.com

Financiamiento:

Ninguno.

Conflicto de intereses:

Ninguno que declarar.

Recibido: 2-6-2015

Aceptado: 21-9-2015

\section{RESUMEN}

En Argentina, las picaduras de alacrán (escorpión) y la mortalidad asociada aumentaron en los últimos años. Durante el período 20012012, se registraron 73617 accidentes y 30 óbitos, casi todos en pacientes pediátricos. A diferencia de otras latitudes del país, en la Ciudad de Buenos Aires y su Conurbano, no se registraron envenenamientos graves o muertes por estos arácnidos. El objetivo fue describir la distribución temporal y geográfica de los hallazgos de Tityus trivittatus en la Ciudad de Buenos Aires desde el 10/1/2001 al 31/12/2012 y su relación con la distribución de los centros asistenciales. Hubo 385 consultas con identificación de escorpiones. Los registros anuales mostraron una tendencia creciente. El georreferenciamiento mostró áreas con mayor frecuencia de hallazgos y densidad en proximidades de ciertas estaciones de ferrocarril y subterráneos, principalmente al este de la ciudad con expansión hacia el oeste. Si bien los centros con servicios de Toxicología coinciden geográficamente con estas zonas, la accesibilidad a centros que disponen del antiveneno puede dificultar su aplicación en el tiempo recomendado. Se sugieren medidas para prevenir demoras en los tratamientos.

Palabras clave: escorpiones, Tityus, mapeo geográfico, envenenamiento, antivenenos.

\section{ABSTRACT}

Scorpion stings and their associated mortality increased in the last years in Argentina, with a cumulative record of 73,617 cases and 30 deaths during the period 2001-2012, occurring almost all the deaths in pediatric patients. However, deaths due to severe envenoming by scorpion stings have not been recorded in Buenos Aires city and suburban regions, although the presence of scorpions in this city has been increasingly reported. We studied the temporal and geographical distribution of Tityus trivittatus findings in Buenos Aires city from the database of the Research and Development Area from the National Institute for Production of Biologics of the National Ministry of Health during the period $10 / 01 / 2001$ to $31 / 12 / 2012$ in order to correlate these findings with the distribution of health centers in the city. In this period 385 consults with identification of scorpions were recorded. Annual records showed a growing trend. Georeferenced data showed that findings appeared to increase in the surroundings of metro and train stations, mainly at the east of the city with expansion to the west. Although Toxicology services are geographically related to the zones with higher density of finding of scorpions, the accessibility to the centers with antivenom may hinder its application in the recommended time; some measures to avoid possible delays in the application of the treatment are suggested.

Key words: scorpions, Tityus, georeferencing, poisoning, antivenoms.

http:/ /dx.doi.org/10.5546/aap.2016.77

\section{INTRODUCCIÓN}

Los accidentes por escorpiones son un problema sanitario en muchas regiones del mundo. ${ }^{1}$ Son la causa estimada de 1500000 casos y 2600 muertes anuales. ${ }^{2,3}$ De las más de 1500 especies de escorpiones conocidas, solo la picadura de algo más de 30 representa un riesgo para la salud humana. ${ }^{4}$ Las estadísticas de diversos países muestran que solo el $10 \%$ de las picaduras de escorpiones provocan envenenamientos y serían responsables de alteraciones generales en los seres humanos. ${ }^{1}$ Esto último es congruente con observaciones realizadas en Argentina en cuanto al uso de antiveneno respecto a las notificaciones de accidentes. ${ }^{5}$ Aunque la mayor cantidad de picadas mundialmente se producen en jóvenes y adultos, la mortalidad pediátrica es diez veces mayor. $^{3}$

En la Argentina, existen alrededor de 60 especies de escorpiones, divididas en dos familias, la Bothriuridae y la Buthidae, dispersas en casi todo el país. ${ }^{6,7}$ Solo unos pocos alacranes, pertenecientes 
todos a la familia Buthidae y, específicamente, al género Tityus, son responsables de los casos de envenenamiento humano. Las diferentes especies de Tityus moran, sobre todo, en las provincias del centro y norte del país ${ }^{7,8}$ y las especies relacionadas con casos de óbitos en Argentina son T. trivittatus ${ }^{9}$ y T. confluens. ${ }^{10} \mathrm{Sin}$ embargo, también hay dos especies cuya picada podría producir accidentes graves e, inclusive, la muerte: T. bahiensis (especie muy común en Brasil y ya existente en Argentina desde hace muchos años) y $T$. serrulatus (cuya aparición se ha descrito recientemente en el noreste del país). Estos dos escorpiones son responsables de envenenamientos humanos en Brasil y el último de ellos es el de principal importancia sanitaria en ese país. ${ }^{11,12}$

En Argentina, se registran anualmente en promedio más de 7000 casos de picadura de alacrán, que representan cerca del $70 \%$ de los accidentes por animales venenosos que se comunican al nivel central, mientras que las muertes causadas por envenenamiento escorpiónico aumentaron en los últimos años. ${ }^{13-15}$ Se han registrado 33 muertes desde 1993, todas en pacientes pediátricos, en su gran mayoría (96\%), menores de 10 años. ${ }^{13}$ A diferencia de los óbitos, la comparación estadística de los datos de accidentes recolectados en los últimos años con los registrados en períodos anteriores no es posible dada la deficiente calidad de los registros en años anteriores.

Tityus trivittatus es el escorpión con mayor importancia médica en Argentina ${ }^{8,9} \mathrm{y}$, en los últimos años, en la Ciudad Autónoma de Buenos Aires (CABA), observamos un aumento tanto del número de hallazgos como del área en que estos se produjeron, lo que comunicamos previamente. ${ }^{16-18}$ Este aumento de hallazgos podría señalar la mejoría del sistema para recoger y registrar los casos y/o indicar una mayor probabilidad de contacto hombre-escorpión y, por lo tanto, un mayor riesgo de ocurrencia de envenenamientos.

Sin embargo, es llamativo que, a diferencia de lo que se observa en otras latitudes del país, a pesar de registrarse accidentes, en la CABA, no se han comunicado, hasta la fecha, envenenamientos graves o muertes por la picadura de T. trivittatus u otra especie de escorpión. Esto, posiblemente, está relacionado con que el veneno de los ejemplares de esta especie capturados en la CABA mostró, hasta el momento, una potencia tóxica experimental menor a la de ejemplares de la misma especie capturados en otras partes del país. ${ }^{19}$ No obstante, recientemente, se produjo un caso que requirió tratamiento en la $\mathrm{CABA}^{20}{ }^{20}$ lo que debe alertar respecto a la potencial toxicidad del veneno de estos escorpiones en la ciudad.

Para la aplicación de medidas preventivas y la toma de acciones, es fundamental conocer la distribución de estos animales. En este trabajo, describimos y analizamos la distribución temporal y geográfica de los registros confirmados de hallazgo de T. trivittatus de la CABA y registrados en el Área Investigación y Desarrollo del Instituto Nacional de Producción de Biológicos, durante el período del 10/1/2001 al 31/12/2012, y ampliamos la información comunicada parcialmente en trabajos previos. ${ }^{16-18,21}$

El objetivo de este trabajo fue, debido a sus implicancias sanitarias, analizar los datos disponibles sobre hallazgos y distribución de T. trivittatus y su relación con los centros de atención toxicológica y con los que disponen del antiveneno escorpiónico específico a fin de evaluar posibles medidas preventivas y estrategias terapéuticas referidas a la accesibilidad a dichos centros.

\section{MATERIALES Y METODOLOGÍA}

Se analizaron los hallazgos de escorpiones recibidos por denuncia espontánea en el Área Investigación y Desarrollo del Instituto Nacional de Producción de Biológicos de la Administración Nacional de Laboratorios e Institutos de Salud "Dr. Carlos G. Malbrán" desde el 1 de enero de 2001 hasta el 31 de diciembre de 2012.

En todos los casos, los escorpiones fueron identificados por un profesional universitario calificado para la identificación de artrópodos venenosos. Se registró la fecha de hallazgo, cantidad de animales, dirección, ambiente y nivel del piso en que fueron hallados. Se calcularon las frecuencias de hallazgo anuales de T. trivittatus y, de acuerdo con los registros, se georreferenciaron los hallazgos reportados que cumplieron con todos los requisitos para poder ser considerados.

Se georreferenció cada notificación y se vinculó el dato de la fecha de hallazgo. Las notificaciones fueron, así, modeladas como puntos geográficos. Se crearon luego áreas de influencia (conocidas como áreas buffer) de tipo concéntricas y con un radio de anillo de 250 metros. Si dos o más casos ocurrieron a menos de 250 metros, las áreas de influencia concéntricas se superpusieron, fusionaron y crearon una nueva área, de manera tal que todos los casos se localizaron a una 
distancia menor de 250 metros del límite del área de influencia. Esta nueva área así creada tiene una forma característicamente "alveolar" resultante de la fusión de áreas circulares superpuestas.

Se seleccionaron los casos según la fecha de registro en rangos acumulativos bianuales (20012002, 2001-2004 hasta 2001-2012).

Se generó luego un mapa de cobertura de áreas de influencia (áreas buffer circulares fusionadas en casos de proximidad) y se identificó el número de casos en cada una de ellas, como así también la extensión en kilómetros cuadrados de estas áreas resultantes.

Para la realización de los análisis, se utilizó el software GIS Geomedia. Para otros estudios estadísticos, se utilizó el método de chi cuadrado y el método de Mann-Whitney (diferencias de hallazgos y diferencias en animales hallados por domicilio entre los sexenios 2001-2006 y 20072012) o análisis de regresión (tendencia de los hallazgos en el período de estudio). Se utilizó el software Prism 5.0 (Graph Pad CA).

Se ubicaron los hospitales públicos de la CABA y se identificaron los Centros de Información, Asesoramiento y Asistencia Toxicológica (CIAAT) con atención de 24 horas los 365 días del año y aquellos que poseían antiveneno escorpiónico.

\section{RESULTADOS DEL ANÁLISIS}

Se registraron 385 hallazgos domiciliarios totales de $T$. trivittatus, que sumaron 470 ejemplares. Los registros de hallazgos anuales desde 2001 mostraron una tendencia creciente. En 51 oportunidades (13\% de los reportes), se encontró más de 1 escorpión por unidad habitacional. El máximo de animales hallados en 1 sola vivienda fue de 11 . En general, se hallaron
1,2 escorpiones por vivienda $y$, considerando aquellas en que se identificó más de un animal, el promedio fue de 2,7 y la mediana de 2 animales con los percentiles de $25 \%$ y $75 \%$ de 2 y 3 , respectivamente (Tabla 1 ).

Los hallazgos georreferenciados $(n=295)$ y su evolución, así como las zonas de la ciudad comprendidas sumadas al área de influencia (superficie aproximada en kilómetros cuadrados en que se hallaron ejemplares: $14 \mathrm{~km}^{2}$ ) y las notificaciones por kilómetro cuadrado (la densidad), se muestran en la Figura 1.

Las áreas con mayor cantidad de hallazgos, en su mayoría, se superponen con las áreas más pobladas y las más transitadas de la ciudad, dada la densidad de terminales ferroviarias y de subterráneos que allí se encuentran y la intensa actividad comercial y administrativa que en ellas se desarrolla (Figura 2).

Se ubicaron los hospitales y los CIAAT con guardias de 24 horas los 365 días del año, que son el Hospital de Agudos "Juan A. Fernández" y el Hospital de Niños "Ricardo Gutiérrez" en la zona norte y el Hospital de Niños "Pedro de Elizalde" ("Casa Cuna") y el Hospital "Francisco Javier Muñiz" en la zona sur. Este último es el único hospital de la ciudad que posee reservas de antiveneno escorpiónico en su farmacia (Figura 3).

Durante todo el período de estudio, la mayor cantidad de hallazgos se produjo en los meses cálidos, entre octubre y abril, lo que representó el $83 \%$ de los hallazgos totales anuales. Se observó durante noviembre-febrero la mayor cantidad de hallazgos ( $57 \%$ del total).

Se encontraron diferencias en la cantidad de hallazgos entre ambos sexenios: 150 animales en el período 2001-2006 y 320 durante el período

TABla 1. Se indican las unidades de vivienda que comunicaron hallazgos, la cantidad de animales hallados y sus frecuencias acumuladas, así como la cantidad de escorpiones comunicados por vivienda.

\begin{tabular}{|c|c|c|c|c|c|}
\hline Años & Casas con hallazgos & Acumulados & Animales hallados & Acumulados & Animales por vivienda \\
\hline 2001 & 16 & 16 & 16 & 16 & 1 \\
\hline 2002 & 11 & 27 & 12 & 28 & 1,1 \\
\hline 2003 & 23 & 50 & 30 & 58 & 1,3 \\
\hline 2004 & 32 & 82 & 44 & 102 & 1,4 \\
\hline 2005 & 18 & 100 & 21 & 123 & 1,2 \\
\hline 2006 & 25 & 125 & 27 & 150 & 1,1 \\
\hline 2007 & 35 & 160 & 40 & 190 & 1,4 \\
\hline 2008 & 31 & 191 & 32 & 222 & 1 \\
\hline 2009 & 58 & 249 & 79 & 301 & 1,4 \\
\hline 2010 & 47 & 296 & 53 & 354 & 1,1 \\
\hline 2011 & 49 & 345 & 65 & 419 & 1,3 \\
\hline 2012 & 40 & 385 & 51 & 470 & 1,3 \\
\hline
\end{tabular}


2007-2012. La diferencia fue significativa ( $p$ 0,009; Mann-Whitney U 2,0) y la tendencia anual de hallazgos estudiada por regresión lineal fue positiva y creciente $\left(\mathrm{r}^{2} 0,48, p 0,0125\right)$. Fue especialmente importante este aumento en los últimos años. No se vio un aumento significativo

FIGURA 1. Hallazgos de T. trivittatus durante el período 2001-2012
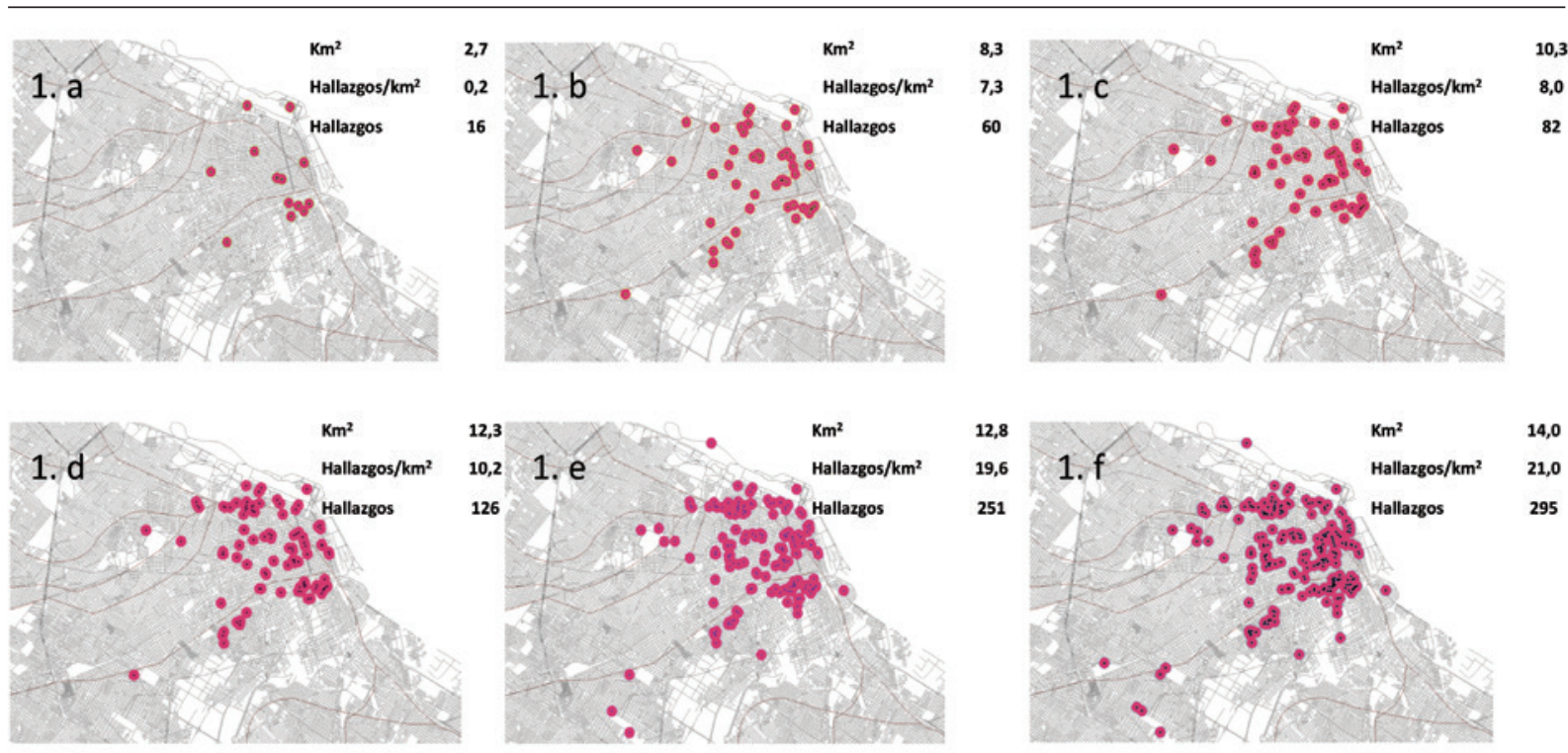

Los cuadros muestran los hallazgos acumulados de ejemplares de T. trivittatus durante períodos bianuales. 1.a: 2001-2002; 1.b: 2001-2004; 1.c: 2001-2006; 1.d: 2001-2008; 1.e: 2001-2010; 1.f: 2001-2012. En cada bienio, se indican los kilómetros cuadrados comprometidos, los hallazgos acumulados y los hallazgos por kilómetro cuadrado. Nótese un aumento de todas las variables en los períodos de estudio. Los datos de las figuras de 1.a a 1.e fueron previamente publicados en Toxins (Basel) 2014; 6: 1434-52.

FIGURA 2. Relación entre hallazgos de escorpiones, densidad de población y red ferroviaria y de subterráneos

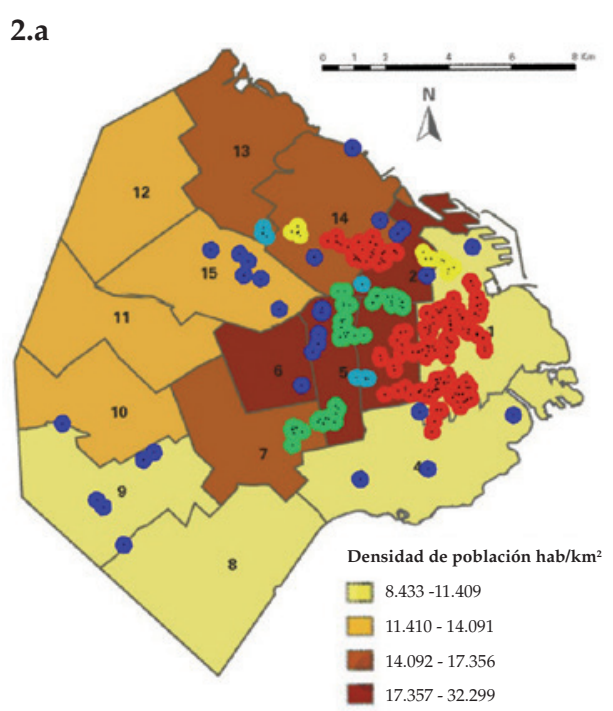

2.b

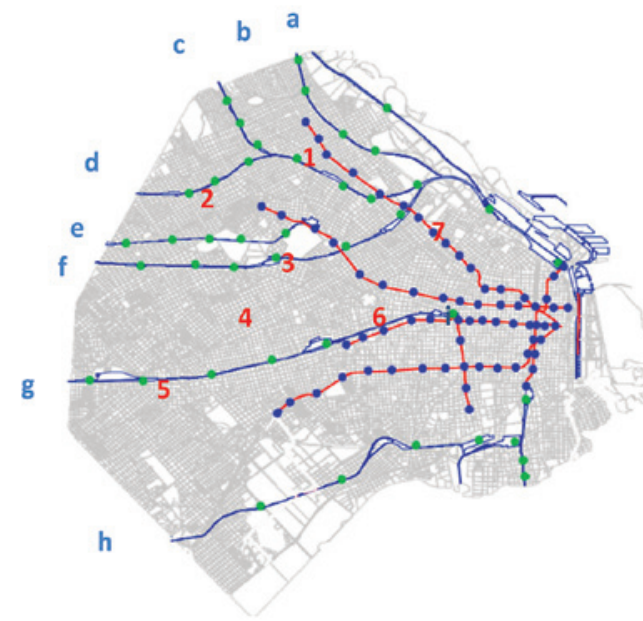
a. FC Belgrano
b, c, d. FC Mitre
e. FC Urquiza
f. FC San Martín
g. FC Sarmiento
h. FC Belgrano Sur
i. FC Roca
1. Subte D
2. Subte B
3. Subte A
4. Subte E
5. Premetro
6. Subte H
7. Subte C

Figura 2.a. Muestra la distribución y la densidad de hallazgos de escorpiones. Áreas buffer definidas por entornos de 250 metros de radio sobre cada hallazgo georreferenciado y fusionadas automáticamente en los casos de superposición. El mapa temático indica el rango de hallazgos en cada área obtenida con un código de color que señala lo siguiente: rojo: entre 30 y 87 hallazgos; verde: entre 10 y 29 hallazgos; amarillo: entre 6 y 9 hallazgos; celeste: entre 4 y 5 hallazgos; azul: entre 1 y 3 hallazgos, superpuestos todos estos en un mapa que indica la densidad de población en la CABA (http://www.buenosaires.gob.ar/areas/hacienda/sis_estadistico/ anuario_general/archivos/mapa1_1a.jpg) al 1 de julio de 2008. Los números muestran las comunas de la CABA.

Figura 2.b. Estaciones de trenes, metro y subterráneo y tendido de vías férreas. Los puntos azules indican las estaciones de subterráneo y metro y los verdes, las estaciones de trenes. 
en la cantidad de animales hallados por domicilio durante los dos sexenios ni entre la cantidad de domicilios con hallazgos múltiples $(p>0,074$; chi cuadrado $=3,18)$ ni en la cantidad de animales por domicilio ( $p$ 0,515; Mann-Whitney U 13,57).

\section{COMENTARIOS}

En estudios anteriores, observamos la distribución de los hallazgos de T. trivittatus en la CABA y su relación con las zonas más pobladas de la ciudad, con las redes de trenes subterráneos y las estaciones de trenes, el hallazgo a diferentes niveles (desde subsuelos hasta en el piso 13) y la falta de asociación con los arroyos subterráneos de la ciudad. ${ }^{18}$ En este trabajo, ampliamos el período de estudio y observamos que la tendencia creciente se mantuvo tanto en el número de hallazgos como en lo referente al aumento del área en que estos se produjeron, según puede observarse en las Figuras 1 y 2. Es importante destacar esto dado que, durante el año 2012, por modificaciones institucionales en el Instituto Nacional de Producción de Biológicos, no se pudo mantener la mecánica de registro histórica de consultas. Los autores fuimos conscientes de que experimentamos un ligero subregistro en el último trimestre de ese año que se fue profundizando, por lo que no consideramos los datos posteriores ya que hubiesen sesgado las observaciones. Sin embargo, dada la tendencia creciente que claramente se ve en el hallazgo de escorpiones y el área de la ciudad comprometida, que también va en ascenso, consideramos importante transmitir la información confiable disponible en relación con los recursos sanitarios de la ciudad para enfrentar esta problemática. Debido a que esta institución es la que recibe mayor cantidad de consultas por temas referidos a escorpionismo en la CABA y la que más datos podría aportar respecto a la dinámica de los hallazgos y su distribución, esta situación debería mejorarse para poder continuar teniendo información precisa y confiable sobre el hallazgo de estos arácnidos en la CABA.

Debe remarcarse que estos datos fueron por consultas espontáneas realizadas a un solo centro. Datos no publicados indican que, en las zonas de alto hallazgo de escorpiones, mediante la búsqueda activa, pueden encontrarse cantidades muy importantes de $T$. trivittatus, lo que no se considera en este trabajo, pero que indica que la cantidad de estos escorpiones es muy importante en las zonas de alta frecuencia de hallazgo y de denuncia espontánea (De Roodt et al., datos sin publicar).

La mayor cantidad de hallazgos se ha registrado en las zonas más antiguas de la ciudad, en coincidencia con lo citado por Maury ${ }^{22}$ y

Figura 3. Distribución de hospitales municipales en la Ciudad de Buenos Aires.

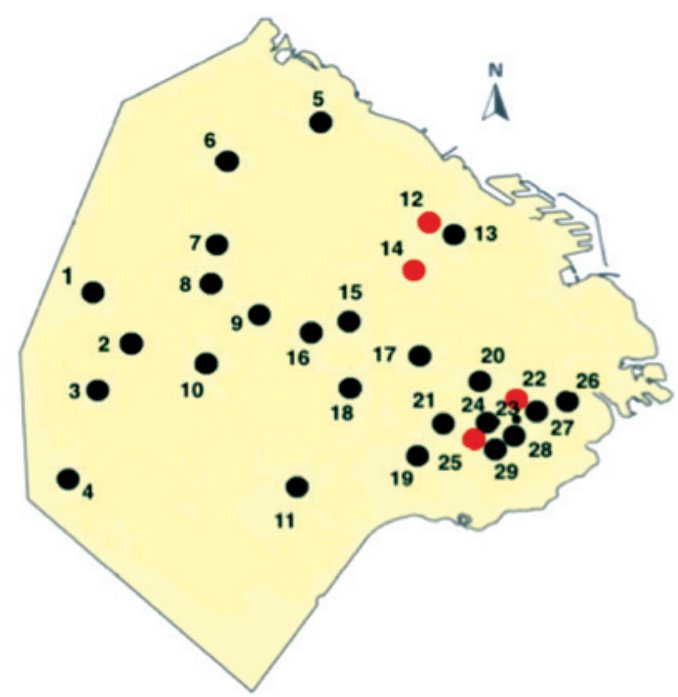

Los diferentes hospitales se muestran en puntos negros. Como puntos rojos, se indican los hospitales con guardias de Toxicología de 24 h, los hospitales Fernández (12), Gutiérrez (14) y Elizalde (22), y el Hospital Muñiz (25) que es el que posee antiveneno. Zubizarreta (1), Roca (2), Vélez Sarsfield (3), Santojani (4), Rehabilitación Psicofísica (5), Pirovano (6), Tornú (7), Alvear (8), Lagleyze (9), Álvarez (10), Piñeiro (11), Fernández (12), Rivadavia (13), Gutiérrez (14), María Curie (15), Durand (16), Ramos Mejia (17), Quemados (18), Penna (19), Santa Lucía (20), Sardá (21), Elizalde (22), Tobar García (23), Udaondo (24), Muñiz (25), Argerich (26), María Ferrer (27), Borda (28), Moyano (29). 
otros, ${ }^{6,16-18}$ en las de mayor densidad poblacional y en aquellas por las que transita la mayor cantidad de personas por razones laborales. En efecto, en las zonas comprendidas alrededor de las estaciones terminales de trenes y subterráneos, hay un tránsito muy intenso de personas, en las mismas zonas que se comunican más hallazgos de T. trivittatus en esta ciudad. Además, tal como se comunicó previamente, los hallazgos siguen un cierto patrón en relación con el tendido de líneas de subterráneo, ${ }^{6}$ en concordancia con lo sugerido por Maury ${ }^{22}$ y observado por nosotros recientemente ${ }^{16-18}$ (Figura 2).

Los CIAAT con guardias físicas de 24 horas los 365 días, así como el único hospital con existencia de antiveneno escorpiónico en su farmacia, se encuentran en la zona de mayor frecuencia de hallazgo de la CABA (Figuras 2 y 3).

Sin embargo, en este punto, debe considerarse un importante aspecto logístico. Dada la rápida absorción de las toxinas de los venenos de escorpiones, ${ }^{11,10,23-26}$ el tratamiento debe aplicarse con la máxima velocidad. Si bien se posee personal entrenado en el Hospital Muñiz y, en su farmacia hay stock de antivenenos, si el accidente se produce en la zona norte de la CABA, el acceso del paciente o el transporte del antídoto puede retrasarse. Este retraso puede ser muy importante si el transporte-traslado se realiza en un día laboral y en las horas pico, lo que consume minutos valiosos durante los cuales las toxinas del veneno se distribuirán para fijarse en los tejidos y ejercerán su efecto. Las recomendaciones del Ministerio de Salud indican la aplicación del antiveneno antes de las dos horas de haber sucedido el accidente, debido a que, tras ese tiempo, la mayoría del veneno se habría fijado en los tejidos y disminuido la capacidad terapéutica de los antivenenos, que es máxima cuando el veneno aún está circulando en la sangre. ${ }^{8}$ Si se considera el tiempo que tarda el paciente en llegar a la consulta y el traslado de este a un centro con antiveneno o del antiveneno al centro donde el paciente esté internado, puede superarse ampliamente el tiempo recomendado. Esta situación puede darse con facilidad si el paciente proviene del norte o el oeste de la CABA. Por ese motivo, sería provechoso y estratégico, en vista de la distribución de los escorpiones en la ciudad y, por lo tanto, de la probabilidad mayor de accidentes en las zonas con mayor cantidad de escorpiones, que hubiese un establecimiento adicional en la zona norte en que se pudiese contar con el antiveneno. Si la tendencia se mantiene, también debería considerarse la zona oeste de la CABA, aunque, en este caso, se cuenta con el Centro Nacional de Intoxicaciones del Hospital Posadas (sobre la Autopista del Oeste, a 5 minutos de la $C A B A$ ), que posee guardias continuas y antivenenos específicos en su banco de antivenenos y rápido acceso desde el oeste de la CABA.

Esto facilitaría tanto el tratamiento con antiveneno de los pacientes que allí concurran como, eventualmente, su provisión al centro que atienda al paciente accidentado. Los hospitales Fernández y Gutiérrez reúnen las características ideales para esto, dado que ambos poseen Servicios de Toxicología con atención de 24 horas durante todo el año y se ubican en la zona norte de la CABA, en la zona de mayor densidad de escorpiones y de población humana.

La toxicidad del veneno de T. trivittatus en la CABA, hasta la fecha, mostró ser muy baja cuando se la compara con la de ejemplares de otras regiones de Argentina. ${ }^{14}$ Sin embargo, en los últimos años, se produjo un caso que requirió tratamiento. ${ }^{20}$ Aunque, hasta ahora, sería este el único caso que presentó signos de envenenamiento moderado en la CABA, hay que tener presente que, en otras ciudades de Argentina en las que históricamente las picadas de escorpiones solo causaban signos locales, en los últimos años, se comenzaron a observar cuadros moderados y graves y hasta se produjeron óbitos, como en las ciudades de Rosario, Santa Fe, Paraná, Catamarca y La Rioja, entre otras. ${ }^{18,27}$

Es necesario recordar que el único tratamiento específico comprobado para tratar los envenenamientos por escorpiones es la aplicación del antiveneno ${ }^{8,20}$ y que la mortalidad por estos envenenamientos se ve reducida diez veces cuando se combina el uso de antiveneno y la internación del niño picado en la Unidad de Terapia Intensiva. ${ }^{8,12,28,29}$ De esto último se desprende la necesidad de contar no solo con el antiveneno específico y con el personal calificado para el diagnóstico y tratamiento, sino también con la disponibilidad de medios adecuada para tratar al paciente que desarrolla un cuadro de envenenamiento, como una unidad de terapia intensiva pediátrica con camas disponibles, adecuada y funcional.

$\mathrm{Si}$ bien los accidentes por escorpiones en la CABA comunicados al nivel central son muy escasos (se han informado, en el período 20132014, solamente 12 casos según datos del Servicio Nacional de Vigilancia en Salud extraídos de 
las planillas C2), el hallazgo de estos arácnidos parece mostrar un patrón creciente, lo que, debido a su potencial peligrosidad, debe alertar a las autoridades sanitarias en cuanto a las medidas por instaurar ante un posible caso de envenenamiento moderado o grave que necesite de la aplicación del antiveneno e internación en una unidad de cuidados intensivos.

Los estudios que se han realizado y se realizan sobre el veneno de estos escorpiones muestran que el veneno procedente de animales de la CABA presenta una toxicidad baja, que, hasta el momento, no se ha modificado, por lo que es importante continuar la evaluación periódica del estudio de la toxicidad en modelos experimentales. Sin embargo, esto solo no alcanza para tomar acciones ante una posible variación de la toxicidad de estos alacranes. El registro sistemático de los hallazgos de estos arácnidos y de los accidentes que se produzcan es necesario para obtener información sobre las zonas de mayor riesgo de contacto hombre-alacrán $\mathrm{y}$, de esa manera, poder tomar las medidas preventivas necesarias ante la posible aparición de casos que requieran la intervención médica. Para esto, se debe contar con bases de datos actualizadas y confiables que involucren las distintas instituciones que reciben consultas por alacranismo o estos arácnidos para su identificación, a fin de tener mayor información de su distribución y movimiento poblacional.

\section{REFERENCIAS}

1. Khattabi A, Soulaymani-Bencheikh R, Achour S, Salmi LR. Classification of clinical consequences of scorpion stings: consensus development. Trans R Soc Trop Med Hyg 2011;105(7):364-9.

2. Chippaux JP, Goyffon M. Epidemiology of scorpionism: a global appraisal. Acta Trop 2008;107(2):71-9.

3. Chippaux JP. Emerging options of the management of scorpion stings. Drug Des Devel Ther 2012;6:165-73.

4. Bücherl W. Classification, biology, and venom extraction of scorpions. En: Bücherl W, Buckley E, eds. Venomous Animals and their Venoms. Volume III: Venomous Invertebrates. New York: Elsevier; 1971.Págs.317-71.

5. De Roodt AR. Estudio del veneno de algunos escorpiones de importancia médica de la Argentina [Tesis de Maestría]. Buenos Aires: Universidad Nacional de San Martín; 2009. 228 pp.

6. Ojanguren-Affilastro AA. Estudio monográfico de los escorpiones de la República Argentina. Rev Iber Aracnol 2005;11:75-241.

7. De Roodt AR, Lanari LC, Laskowicz RD, Costa de Oliveira V. Identificación de los escorpiones de importancia médica en la Argentina. Acta Toxicol Argent 2014;22(1):5-14.

8. Argentina. Ministerio de Salud de la Nación. Guía de prevención, diagnóstico, tratamiento y vigilancia epidemiológica delenvenenamiento por escorpiones. Buenos Aires: Ministerio de Salud; 2011.

9. De Roodt AR, García SI, Salomón OD, Segre L, et al. Epidemiological and clinical aspects of scorpionism by
Tityus trivittatus in Argentina. Toxicon 2003;41(8):971-7.

10. De Roodt AR, Lago NR, Salomón OD, Laskowicz RD, et al. A new venomous scorpion responsible for severe envenomation in Argentina: Tityus confluens. Toxicon 2009;53(1):1-8.

11. Cupo P, De Azevedo-Marques MM, Hering SE. Escorpionismo. Em: Costa Cardoso JL, Siqueira França FO, Wen FH, Saint Ana Málaque CM, et al, eds. Animais Peçonhentos no Brasil: biologia, clínica eterapêutica dos acidentes. São Paulo: Sarvier; 2003.Pags.198-208.

12. Brasil. Ministério da Saúde. Manual de Diagnóstico e Tratamento de Acidentes por Animais Peçonhentos. Brasilia: Fundação Nacional de Saúde; 1992.

13. Casas N, Geffner L, Echenique H, Costa de Oliveira $\mathrm{V}$, et al. Epidemiologic situation of envenomation by venomous animals in Argentina. 2007-2011 period. Toxicon 2012;60(2):238-9.

14. De Roodt AR, Oliveira V, de Pietri D, García S. Accidentes por animales venenosos comunicados al Ministerio deSalud de la Nación en el período 2005-2009. Acta Toxicol Argent 2010;18(Supl):63.

15. De Roodt AR, Lanari LC, García SI, Costa de Oliveira V, et al. Accidentes y óbitos por envenenamiento por animales venenosos-ponzoñosos en Argentina en el período 20002011. Acta Toxicol Argent 2013;21(Supl):100.

16. Laskowicz R, Scarlato E, Lanari L, Blanco G, et al. Localización geográficadeejemplaresdeTityustrivittatushalladosenlaciudad de Buenos Aires. Acta Toxicol Argent 2011;19(Supl):94-95.

17. Blanco G, LaskowiczRD,ScarlattoE, Casas N, etal.Increased incidence of Tityus trivittatus envenoming in the City of Buenos Aires. Toxicon 2012;60(2):188.

18. De Roodt AR. Comments on environmental and sanitary aspects of the scorpionism by Tityus trivittatus in Buenos Aires City, Argentina. Toxins (Basel) 2014;6(4):1434-52.

19. De Roodt AR, Coronas FI, Lago N, González ME, et al. Generalbiochemical and immunological characterization of the venom from the scorpion Tityus trivittatus of Argentina. Toxicon 2010;55(2-3):307-19.

20. Docampo PC, Fernández ME. Escorpionismo: presentación de un posible caso grave ocurrido en la Ciudad Autónoma de Buenos Aires. Acta Toxicol Argent 2011;19(1):16-8.

21. Salomón OD, De Roodt AR. Escorpiones: denuncia espontánea en dos centros de referencia en la ciudad de Buenos Aires, 1997-2000. Medicina (B Aires) 2001;61(4):391-6.

22. Maury EA. Tityus trivittatus en la Argentina, nuevos datos sobre distribución, partenogénesis, sinantropía y peligrosidad (escorpiones, buthidae). Publicaciones de extensión cultural y didáctica del Museo Argentino de Ciencias Naturales "Bernardino Rivadavia" 1997;24:1-24.

23. De RezendeNA, Dias MB, Campolina D, Chavéz-Olórtegui C, et al.Standarization of an enzyme linked immunosorbent assay (ELISA) for detecting circulating toxic venom antigens in patients stung by the scorpion Tityus serrulatus. Rev Inst Med Trop Sao Paulo 1995;37(1):71-4.

24. De Rezende NA, Chavéz-Olórtegui C, Amaral CF. Is the severity of Tityus serrulatus scorpion envenoming related to plasma venom concentrations? Toxicon 1996;34(7):820-3.

25. Ismail M, Abd-Elsalam MA. Are the toxicological effects of scorpion envenomation related to tissue venom concentration? Toxicon 1988;26(3):233-56.

26. Santana GC, Freire AC, Ferreira AP, Cháves- Olórtegui C, et al. Pharmacokinetics of Tityus serrulatus scorpion venom determined by enzyme-linked immunosorbent assay in the rat. Toxicon 1996;34(9):1063-6.

27. Piola JC, Prada DB, Waksman JC, Evangelista M. Increase mortality and morbidity of Tityus trivitattus envenomations in Argentina. Clin Toxicol (Phila) 2006;44:650.

28. Freire-Maia L, Campos JA, Amaral CF. Approaches to the treatment of scorpionenvenoming. Toxicon 1994;32(9):1009-14.

29. Freire-Maia L, Campos JA. On the treatment of the cardiovascular manifestations of scorpion envenomation. Toxicon 1987;25(2):125-30. 